\title{
How do Maternal PTSD and Alexithymia Interact to Impact Maternal Behavior?
}

\author{
Daniel S. Schechter • Francesca Suardi - Aurelia Manini - Maria Isabel Cordero • \\ Ana Sancho Rossignol · Gaëlle Merminod • Marianne Gex-Fabry • \\ Dominik A. Moser $\cdot$ Sandra Rusconi Serpa
}

Published online: 10 July 2014

(C) Springer Science+Business Media New York 2014

\begin{abstract}
Maternal interpersonal violence-related posttraumatic stress disorder (IPV-PTSD) is known to be associated with impairment of a mother's capacity to participate in mutual emotion regulation during her child's first years of life. This study tested the hypothesis that maternal difficulty in identifying feelings in self and other, as an important dimension of the construct of alexithymia, together with maternal IPV-PTSD, would be negatively associated with maternal sensitivity. Maternal sensitivity to child emotional communication is a marker of maternal capacity to engage in mutual regulation of emotion and arousal. Following diagnostic interviews and administration of the Toronto Alexithymia Scale, 56 mothers and their toddlers (ages 12-42 months) were filmed during free-play and separation/novelty-exposure. Observed maternal sensitivity was coded via the CARE-Index. Maternal IPV-PTSD severity, difficulty in identifying emotions, and lower socio-economic status were all associated with less maternal sensitivity, and also with more
\end{abstract}

D. S. Schechter $\cdot$ F. Suardi $\cdot$ A. Manini

M. I. Cordero - A. S. Rossignol · G. Merminod .

D. A. Moser · S. R. Serpa

Research Unit, Child and Adolescent Psychiatry Service,

University of Geneva Hospitals, Geneva, Switzerland

D. S. Schechter · M. I. Cordero · M. Gex-Fabry

Department of Psychiatry, Geneva University Hospitals, Geneva,

Switzerland

D. S. Schechter $(\square)$

Unité de recherche du service de psychiatrie de l'enfant et de

l'adolescent (SPEA), Rue Verte 2, 1205 Geneva, Switzerland

e-mail: daniel.schechter@hcuge.ch

F. Suardi - D. A. Moser - S. R. Serpa

Department of Psychology, Faculty of Psychology and

Education, University of Geneva, Geneva, Switzerland maternal controlling and unresponsive behavior on the CARE-Index.

Keywords Violence $\cdot$ Post-traumatic stress disorder (PTSD) - Emotion regulation - Alexithymia · Parenting · Infancy and early childhood

\section{Introduction}

Violence exposure is among the most traumatogenic types of life events. Interpersonal violence (IPV) which includes adult physical and/or violent assault in addition to comorbid childhood exposures to physical and/or sexual abuse and/or domestic violence, is associated with a prevalence rate of post-traumatic stress disorder (PTSD) as high as 80-90 \% [1]. The nature and course of interpersonal violence-related PTSD (IPV-PTSD) is often "complex" and chronic [2, 3].

PTSD, regardless of the type of traumatic event, most often affects women of childbearing age; the prevalence ratio of women to men is as high as 3:1 [4]. Given that the demographics of IPV-PTSD include a large number of potential and actual mothers of infants and young children, it is imperative that researchers consider the effects of the disorder on the mother-child relationship and on the socialemotional development of the infant and young child in order to prevent subsequent negative effects on child development.

The portion of childhood during which one refers to the child as "a toddler", ages 1-3 years, is an especially sensitive developmental period during which the child depends on mutual regulation of emotion and arousal with his caregiver. He also depends on his caregiver's capacity to set limits with normative exploratory, instrumental, and 
hostile aggression for healthy social-emotional development and the foundation of self-regulatory capacities that permit the child to enter kindergarten by age $4-5$ years [5, 6]. When maternal psychopathology interferes with maternal availability towards these ends, there is a greater risk for subsequent development of conduct disorder, autoaggressive behavior and other psychopathology that may well contribute to intergenerational transmission of violence and related trauma $[5,7,8]$. This is especially salient when the child's father has been violent and thus the child must depend even more on his attachment to the mother for a sense of security, and as a model for prosocial behavior. The latter can become particularly difficult if the child's mother herself does not feel secure and remains hypervigilant to potential attack, such that she cannot assist her toddler in reading and responding to his emotional communication as is necessary for supporting emotion regulation. Her behavior would thus likely reflect diminished sensitivity $[9,10]$.

Maternal sensitivity was first defined by Mary Ainsworth ([11], p. 2) as "the mother's ability to perceive and to interpret accurately the signals and communications implicit in her infant's behavior, and given this understanding, to respond to them appropriately and promptly". Ainsworth identified four "essential components" of maternal sensitivity that could be measurable by her maternal sensitivity scales: (a) her awareness of the infant's signals; (b) an accurate interpretation of them; (c) an appropriate response to them; and (d) a prompt response to them. Many mothers with demonstrably low sensitivity to their child's emotional communication also had histories of exposures to family violence, abuse, and neglect. Lyons-Ruth and Block [12] first showed that similarly "at-risk" mothers' severity of PTSD symptoms was inversely related to the degree of sensitive caregiving behavior (i.e. intrusive and aggressive behavior towards the child, as well as avoidant and withdrawing behaviors). We set out to replicate and extend their findings in order to understand how IPVPTSD mothers might affect their toddlers and how their toddlers might affect them in terms of mutual regulation of emotion and arousal [13].

IPV-PTSD mothers have reported difficulties with respect to the reading and interpretation of their child's emotional communication [14]. In particular, a confusion between negative emotions involving high-arousal has been noted, namely, a confusion between fear and anger $[13,14]$. This particular form of confusion, we posited could be screened with measures of alexithymia. This idea is further supported by the reported association between alexithymia and complex PTSD [15].

Alexithymia was first described as a personality trait or psychological deficit in which affected individuals have trouble distinguishing and appreciating others, thus leading to less empathic responsiveness [16]. The question has emerged as to whether this apparent trait might, among patients with PTSD, actually represent an aspect of this disorder's emotional numbing and dissociation [17]. Alexithymia has specifically been shown to be associated with emotional numbing as a way of avoiding painful trauma-associated memory-traces among patients with IPV-PTSD and burn- and accident-related PTSD [18, 19]. These findings support the interpretation that alexithymia can represent a psychological consequence of traumatization in some individuals $[18,20]$. Yet studies have also suggested that, independent of any specific form of psychopathology, individuals with alexithymic traits show distinct patterns of neural activity in response both to visual stimuli depicting facial expression as well as auditory stimuli composed of angry, surprised, and neutral prosody [21]. Maternal alexithymia may therefore also represent a pre-morbid or "primary" trait that can be a risk-factor for traumatization and re-traumatization, perhaps linked to an early sensitive developmental period in the context of attachment [22].

Many early disturbances of attachment are frequently comorbid with childhood histories of interpersonal violence exposure, and many women who go on to become victims of interpersonal violence in adulthood have such a history. Because of this, anything short of a prospective longitudinal study would not definitively be able to answer the question as to whether alexithymia is more of a psychological defense in the context of PTSD or a marker of an early attachment disturbance [23].

Recent research supports that specific alexithymic errors in identifying and distinguishing "fear" from "anger" are likely salient and related to the negativity and distortion of maternal attributions that inform empathic maternal responsiveness to her child [24]. Further research is therefore needed in order to examine the link between maternal difficulty identifying emotion and mothers' sensitive caregiving behavior with their toddlers. Given the dearth of literature that examines maternal sensitivity and its relationship to PTSD and alexithymia, we decided to focus on this question in the present study.

We hypothesized the following:

1. The diagnosis and severity of traumatized mothers' posttraumatic stress, in particular, their avoidance/ emotional numbing would be significantly associated with mothers' difficulty in correctly identifying others' emotions (i.e. "feelings").

2. This maternal difficulty would be negatively associated with maternal sensitivity since, given this alexithymic difficulty, one would expect an affected mother to have trouble identifying her child's and her own feelings. 
3. We hypothesized, therefore, that greater maternal difficulty identifying feelings in self and other would together with maternal IPV-PTSD be associated with less maternal sensitivity than either predictor alone.

\section{Methods \\ Participants and Procedures}

The study protocol was approved by the institutional review board of the University of Geneva Hospitals.

Inclusion and exclusion criteria were as follows: Biological mothers were included in the study if they had lived with their child for the majority of the child's life since birth. Due to physiologic measurements taken, women who were pregnant or breast-feeding were not accepted into the study. Children were included in the study if they were 12-42 months of age at the time of scheduled mother-child behavioral observations. Mothers were excluded if they were actively substance abusing or psychotic. Mothers and children were excluded from the study if they were physically and/or mentally impaired in such a way that would interfere with the ability to participate in laboratory tasks (i.e. cognitive, sensory, and motor impairments).

Sixty women and their children were recruited by flyers posted at the University of Geneva Hospitals and Faculties of Medicine and Psychology as well as at community centers, daycares, schools including domestic violence agencies and shelters. All comers were screened. Out of 60 who were screened and provided informed consent, 4 mothers were found to have a full-PTSD diagnosis or clinically significant symptoms (subthreshold) due to a non-IPV traumatic event (i.e. medical-surgical event, accident, natural disaster, etc.) and were thus excluded from the present analyses. Thirty-four mothers had PTSD symptoms, with the "A-Criterion" of the DSM-IV-TR related to IPV, among whom 24 met criteria for DSM-IVTR diagnosis and 10 had clinically significant symptoms that were below the threshold for full-diagnosis. Thus, 56 French-speaking mothers (ages 18-45 years) and children participated (ages 12-42 months) including 34 mothers and children in the PTSD case-group and 22 mothers and children in the non-PTSD group. Fathers and other romantic partners of mothers were not seen in the study given concerns over safety and maintenance of trust for women who had experienced partner violence. Thus data about fathers were obtained by maternal report rather than from the fathers themselves.

Within 1 month after the screening visit, participants completed 2 videotaped visits over the ensuing 1-2 month period Fig. 1 shows the sequence of the screening, 1st and 2nd visits separated by 1-2 weeks. During the screening visit, following informed consent, mothers were given a socio-demographic and life-events interview followed by several self-report questionnaires. During the following visit, mothers were interviewed without their child present, with a focus on the mother's mental representations of her child and relationship with her child, an elaboration of her traumatic life-events, followed by structured diagnostic interviews and a series of dimensional measures. Then, 1-2 weeks later, mothers were asked to bring their child to the lab for a parent-child interaction procedure [25] followed by administration of measures focusing on the child's life events, psychopathology, and social-emotional development. Salivary samples were taken for measurement of cortisol and DNA extraction for analyses that are not discussed in this paper.

After each visit, mothers received 50 Swiss francs along with a small book or toy for their child.

\section{Measures}

\section{Socio-Demographic Variables}

During the screening session we conducted an interview with the mothers using the Geneva Socio-demographic Questionnaire (GSQ; [26]) which was adapted from the Structured Clinical Interview for the DSM-IV [27] and developed for the present study in order to obtain a detailed overview of the parents' socioeconomic status, characteristics and history of the mother-partner relationships, and exposure to stressful life-events (i.e. interpersonal violence, substance abuse, economic difficulties, immigration, and physical and mental health problems and interventions, and child protective and judicial services involvement). The family socio-economic status (SES) was calculated using the Largo Index [28], which is a well-validated SES index used in pediatric research in Switzerland that takes into account both parental educational attainment and occupational status.

\section{IPV and Other Traumatic Life-Events}

History of experience of traumatic events during childhood was assessed via the Brief Physical and Sexual Abuse Questionnaire (BPSAQ; [29]), and supplemented for other events during adulthood with the Traumatic Life Events Questionnaire (TLEQ). The TLEQ assesses 22 life events that could fulfill the "A-Criterion" for the DSM-IV diagnosis. The TLEQ shows stability and convergent validity across various studies and minority populations [30]. Twelve items that asked for the same events as the BPSAQ were eliminated from the TLEQ. Scoring of the BPSAQ was undertaken as described in a previous paper by the first 
Fig. 1 Protocol design

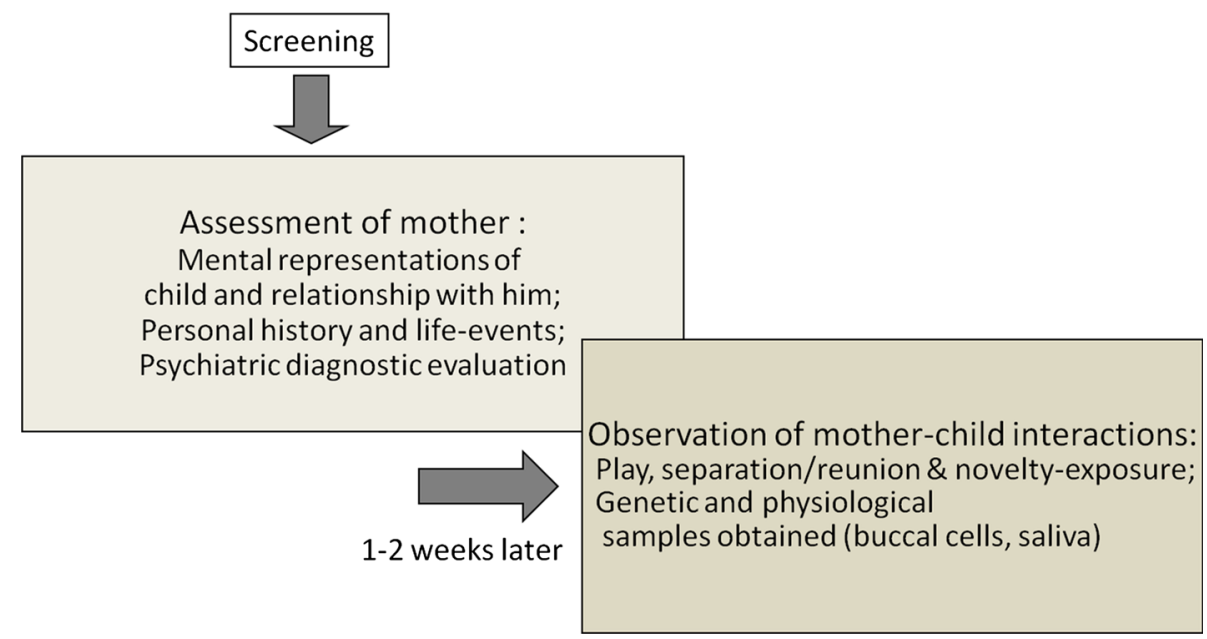

author [10]. The severity of physical violence of the mother's partner and herself in the context of her adult romantic relationships was measured via the Conflicts Tactics Scale 2 Short Version (CTS2; [31]). This wellvalidated measure consists of 20 items that ask about tactics used by the subject's partner and herself in order to resolve relational conflict including physical aggression along a 7-point scale.

\section{Maternal Psychopathology}

During an initial videotaped interview IPV-exposed and non-IPV-exposed mothers underwent a variety of psychometrics including the Clinician administered PTSD scale (CAPS; [32]) to assess lifetime PTSD and the Post-traumatic Symptom Checklist -Short Version (PCL-S) additionally to assess current PTSD symptoms [33]. Subjects on both measures with no IPV and no PTSD symptoms were coded as having the minimum score of 16 . Subjects with IPV exposure but with no PTSD symptoms were coded as having 17. For categorical analyses, mothers met criteria for violence-related PTSD if their A-criterion trauma was of a violent nature (i.e. due to child physical or sexual abuse or family violence exposure and/or adult physical or sexual assault), and if the CAPS score was at or above 55 and the PCL-S score was at or above 40 [34]. Subjects that were subthreshold for diagnosis yet with significant clinical symptoms such that they were included in the IPV-PTSD groups were those who had a CAPS score above 55 and a PCL-S score over 30 (rather than 40 as required for full-diagnosis).

Maternal depressive symptoms were also assessed. Mothers were evaluated via the Beck Depression Inventory-II (BDI-II; [35]) as a self-report measure for the current subjective symptom severity, as well as with the Structured Clinical Interview for the DSM-IV Mood
Disorders Module (SCID; [27]), for lifetime and current diagnosis and number of symptoms as a marker of severity.

\section{Parenting Stress}

Parenting Stress was measured via the Parenting Stress Index-Short Form (PSI-SF; 36). This score includes items related to distress that parents feel in relation to their role as a parent and in light of other personal stressors, as well as parent-child relationship dysfunction, and child behavior that poses difficulty to parents. The PSI-SF has 36 items and each item is assessed on a five-point Likert scale, from 1 (strongly disagree) to 5 (strongly agree). It is a standardized instrument with a validated French translation. The PSI-SF shows high internal consistency (Cronbach's alpha .92) [36].

\section{Alexithymia}

Alexithymia was assessed using the well-validated Frenchlanguage version of the Toronto Alexithymia Scale (TAS20) which consists of 20 items [37]. The TAS-20 and its component subscales demonstrate good internal consistency (Cronbach's alpha .81) and test-retest reliability (.77). Its 3 component subscales are: Difficulty describing feelings; difficulty identifying feelings; externally-oriented thinking [38].

For this study, we used specifically the subscale "Difficulty Identifying Feelings" to test our a priori hypothesis that mothers with higher levels of difficulty in identifying their own and their child's feelings would have lower levels of maternal sensitivity (see "Introduction").

\section{Maternal Behavior}

Maternal sensitivity was measured via structured behavioral observations during 5 min of mother-child play. Two blind 
Table 1 Sociodemographic characteristics and history

$$
\text { Overall }(\mathrm{n}=56) \quad \text { Range } \quad \text { IPV-PTSD }(\mathrm{n}=34) \quad \text { Controls }(\mathrm{n}=22) \quad \text { Mann-Withney }
$$

Z/Chi square [1]

\begin{tabular}{|c|c|c|c|c|c|}
\hline \multicolumn{6}{|l|}{ Demographics } \\
\hline Mothers age (in years) & $34.0(5.7)$ & $22-47$ & $33.1(5.4)$ & $35.3(5.8)$ & -1.33 \\
\hline Child age (in months) & $26.7(8.8)$ & $12-42$ & $26.4(9.1)$ & $27.2(8.6)$ & -.40 \\
\hline Child gender ( $\%$ male $)$ & 51.7 & & 55.6 & 45.4 & .58 \\
\hline Socioeconomic status (low values $=$ better status) & 5.2 & $2-10$ & $5.9(2.2)$ & $4.2(2.0)$ & $-2.59 * * *$ \\
\hline Mother separated or divorced from father & $19.6 \%$ & & $32.3 \%$ & $0 \%$ & $8.86^{* *}$ \\
\hline Mother without partner in home & $21.4 \%$ & & $35.3 \%$ & $0 \%$ & $9.88 * * *$ \\
\hline \multicolumn{6}{|l|}{ Father's history } \\
\hline Alcohol abuse & $13.4 \%$ & & $23.0 \%$ & $0 \%$ & $5.93 *$ \\
\hline Drug abuse & $41.8 \%$ & & $55.9 \%$ & $22.7 \%$ & $4.72 *$ \\
\hline Father violent towards others & $34.5 \%$ & & $65.7 \%$ & $0 \%$ & $24.2 * * *$ \\
\hline \multicolumn{6}{|l|}{ Mother's history } \\
\hline Alcohol abuse & $9.1 \%$ & & $14.7 \%$ & $4.5 \%$ & 1.44 \\
\hline Drug abuse & $25.5 \%$ & & $35.3 \%$ & $13.6 \%$ & 3.20 \\
\hline Mother violent towards others & $33.9 \%$ & & $48.6 \%$ & $13.6 \%$ & $7.24 * *$ \\
\hline Suicide attempts/injuring self & $20.0 \%$ & & $28.6 \%$ & $4.8 \%$ & $4.71 *$ \\
\hline
\end{tabular}

$(p)+\leq .1 ; * \leq .05 ; * * \leq .01 ; * * \leq .005$

raters who were psychologists trained to reliability on the CARE-Index [39] coded the maternal and child behaviors. For the coding of maternal sensitivity, the coding procedure focused these raters' attention on seven aspects of maternal behavior some of which assess affect (facial expression, vocal expression, position and body contact, expression of affection) with other assessing "cognition", i.e., temporal order and interpersonal contingency (pacing of turns, control of the activity, and developmental appropriateness of the activity). Each aspect of behavior was evaluated separately. The scores were then summed to generate the maternal sensitivity scale score. This scale score has a range from 0 to 14 , with zero sensitivity being dangerously insensitive, 7 being normally sensitive, and 14 being outstandingly sensitive. The CARE-Index also provides two additional scales: Controlling (i.e. intrusive) and Unresponsive (i.e. avoidant and withdrawing) maternal behavior that are coded using a similar scale-score to that of maternal sensitivity [40]. While our hypothesis did not concern these two additional scales, we have included them to help characterize maternal behavior associated with maternal IPV-PTSD [41]. Interrater reliability was excellent for all three scales $(\mathrm{ICC}=.86)$. The Infant and Toddler versions of the CARE-Index are wellvalidated $[42,43]$.

\section{Data Analysis}

Group comparisons (mothers with full diagnosis or subthreshold IPV-PTSD vs. non-PTSD mothers) proceeded with Chi square tests for categorical variables and Mann-
Whitney $U$ tests for continuous variables (Tables 1, 2). Associations of continuous measures (e.g. maternal PTSD severity reported as the CAPS score, alexithymia and degree of maternal sensitivity) were analyzed using Pearson correlation coefficients (Table 3). Multiple linear regression models were then used to investigate maternal sensitivity (CARE-Index) as a function of various predictors (Table 4). All analyses were performed using SPSS versions 19 and 22 (IBM, Armonk NY, USA). Significance level was set at .05 (two-tailed tests).

\section{Results}

Among the 56 mothers whose data were analyzed and as shown in Table 1, maternal mean age was 34 years (SD 5.7), and while there were relatively older mothers in the control group, the difference between IPV-PTSD and nonPTSD groups was not significant $(p>.1)$ Children's mean age was 27 months (SD 8.8) at the time of the parent-child interaction visit during which maternal sensitivity was measured, with no significant difference between IPVPTSD and non-PTSD groups $(p>.8)$. Nearly equal numbers of boys and girls participated in the study (29 boys; 27 girls); while there were relatively fewer boys in the control group, this group difference was not significant $(p>.3)$. Group comparisons of additional socio-demographic variables are provided in Table 1 . Of note, mothers with IPVPTSD, had significantly lower socio-economic status (SES). 
Table 2 Comparison of the IPV-PTSD group and the control group with respect to violence by most recent partner and by mother herself in the most recent relationship, PTSD (lifetime and current) and depression (current) symptom severities, overall Parenting Stress score on the PSI-SF and overall Alexithymia score and subscale scores on the TAS-20, as well as maternal sensitivity, controllingness and unresponsiveness (according to the CARE-Index)

\begin{tabular}{|c|c|c|c|c|c|}
\hline & Overall $(\mathrm{n}=56)$ & Range & IPV-PTSD (n = 34) & Controls $(n=22)$ & Mann-Withney Z \\
\hline Violence by partner (CTS) & $2.4(6.3)$ & $0-30$ & $4.2(7.8)$ & $.05(.21)$ & $-3.08 * * *$ \\
\hline Violence by self (CTS) & $.9(2.9)$ & $0-16$ & $1.3(3.6)$ & $.2(.7)$ & $-2.32 * *$ \\
\hline Lifetime PTSD (CAPS) & $62.0(36.3)$ & $16-129$ & $86.4(19.7)$ & $23.4(16.6)$ & $-6.09 * * *$ \\
\hline Current PTSD (PCL-S) & $33.6(15.4)$ & $16-66$ & $44.2(9.4)$ & $16.7(1.2)$ & $-6.36 * * *$ \\
\hline Current depression (BDI) & $9.3(8.0)$ & $0-34$ & $12.7(8.7)$ & $4.4(3.1)$ & $-3.94 * * *$ \\
\hline Parenting stress (PSI-SF) & $40.5(20.9)$ & $12-122$ & $46.7(22.4)$ & $32.3(15.0)$ & $-2.44 * * *$ \\
\hline Alexithymia (TAS-20 overall score) & $44.8(11.6)$ & $22-75$ & $47.4(12.2)$ & $40.55(9.2)$ & $-2.27 *$ \\
\hline Difficulty identifying feelings & $15.0(5.8)$ & $7-27$ & $16.80(6.2)$ & $11.9(3.7)$ & $-3.25 * *$ \\
\hline Difficulty describing own feelings & $12.9(4.6)$ & $5-23$ & $13.4(4.7)$ & $12.05(4.1)$ & -1.16 \\
\hline Outwardly oriented thoughts & $16.8(4.3)$ & $8-25$ & $17.1(4.69)$ & $16.5(3.66)$ & -.33 \\
\hline \multicolumn{6}{|l|}{ CARE-index } \\
\hline Maternal sensitivity & $5.4(1.3)$ & $3-7$ & $4.8(1.3)$ & $6.1(1.1)$ & $-3.26 * *$ \\
\hline Maternal controllingness & $2.8(1.6)$ & $0-7$ & $3.2(1.6)$ & $2.3(1.5)$ & $-2.31 *$ \\
\hline Maternal unresponsiveness & $2.7(1.7)$ & $0-6$ & $3.0(1.7)$ & $2.2(1.5)$ & $-1.74+$ \\
\hline
\end{tabular}

$(p)+\leq 1 ; * \leq 05 ; * * \leq .01 ; * * * \leq .005$

Table 3 Correlations between socioeconomic status, severity of adult IPV, maternal psychopathology, parenting stress, maternal difficulty in identifying feelings, and maternal sensitivity, controllingness, and unresponsiveness on the CARE-Index

\begin{tabular}{|c|c|c|c|c|c|c|c|c|c|}
\hline Variable & 1 & 2 & 3 & 4 & 5 & 6 & 7 & 8 & 9 \\
\hline 1. SES & 1 & $.29 *$ & $.26^{*}$ & .14 & .17 & $.34 * *$ & $-.37 * *$ & .05 & $.39 * * *$ \\
\hline 2. IPV & & 1 & $.30 * *$ & $.28 *$ & .12 & .21 & -.25 & .09 & .20 \\
\hline 3. PTSD & & & 1 & $.57 * * *$ & $.44 * * *$ & $.43 * * *$ & $-.51 * * *$ & $.42 * * *$ & $.22+$ \\
\hline 4. Depression & & & & 1 & $.62 * * *$ & .22 & $-.28 *$ & .09 & .10 \\
\hline 5. Parenting stress & & & & & 1 & $.40 * * *$ & $-.25+$ & .16 & .11 \\
\hline $\begin{array}{l}\text { 6. Difficulty identifying } \\
\text { feelings }\end{array}$ & & & & & & 1 & $-.40 * * *$ & $.23^{*}$ & $.26+$ \\
\hline 7. Maternal sensitivity & & & & & & & 1 & $-.61 * * *$ & $-.47 * * *$ \\
\hline 8. Maternal controllingness & & & & & & & & 1 & -.21 \\
\hline 9. Maternal unresponsiveness & & & & & & & & & 1 \\
\hline
\end{tabular}

Maternal variables: SES socio-economic status, IPV Conflict Tactics Scale Partner Physical Aggression severity in most recent relationship; PTSD CAPS overall score; depression = BDI-II (current); parenting stress = overall PSI-SF score; difficulty identifying feelings = TAS-20 subscale score; maternal sensitivity, maternal controllingness, and maternal responsiveness = CARE-Index score based on observations of play $(p)+\leq .1 ; * \leq .05 ; * * \leq .01 ; * * * \leq .005$

Life events that are typically reported as "traumatic" by mothers were analyzed according to IPV-PTSD versus non-PTSD group-membership, and were significantly more common among IPV-PTSD mothers, yet frequently noted among non-IPV mothers at a higher prevalence than expected (see Fig. 2).

Mothers were also asked if their child's father had experienced family or other interpersonal violence during his own childhood with the understanding that these data would be based on maternal report and subject to error. According to mothers, as shown in Table 1, significantly more case-fathers than control-fathers experienced family or other interpersonal violence during their childhood up to age $16(55.6 \%$ of case-fathers as compared to $30 \%$ of control-fathers, $p<.001)$. The authors did not ask mothers to provide further trauma history data concerning their child's father. Fathers of children with IPV-PTSD mothers as compared to non-PTSD mothers were, in addition to being more violent, more likely to have been substance abusers.

IPV-PTSD mothers showed a higher comorbidity of adult experience of IPV with other forms of violent trauma 
Table 4 Maternal sensitivity as a function of maternal PTSD symptom severity (CAPS), maternal ability to identify feelings in others (TAS-20) and socio-economic status (univariate and multiple linear regression models)

\begin{tabular}{lllllll}
\hline $\begin{array}{l}\text { Predictors of } \\
\text { maternal sensitivity }\end{array}$ & B & SE & $\beta$ & $p$ & $\mathrm{R}^{2}$ & \multicolumn{1}{l}{$\begin{array}{l}\text { Sig } \\
\mathrm{R}^{2} \Delta\end{array}$} \\
\hline 1. PTSD (univariate) & -.02 & .004 & -.51 & $<.001$ & .26 & $\ldots$ \\
2. Difficulty & -.09 & .03 & -.40 & .002 & .16 & $\ldots$ \\
$\begin{array}{l}\text { identifying feelings } \\
\text { (univariate) }\end{array}$ & & & & & & \\
3. SES (univariate) & -.22 & .07 & -.37 & .005 & .14 & $\ldots$ \\
4. Difficulty & -.16 & .03 & -.29 & .036 & .21 & versus \\
identifying feelings & & & & & & $1: .036$ \\
SES & -.06 & .08 & -.28 & .041 & & versus \\
& & & & & & $2: .041$ \\
5. Difficulty & -.05 & .03 & -.22 & .097 & .31 & versus \\
identifying feelings & & & & & & $1: .097$ \\
PTSD & -.015 & .005 & -.43 & .002 & & versus \\
& & & & & & $2: .002$ \\
6. PTSD & -.015 & .004 & -.44 & .001 & .32 & versus \\
& & & & & & $1: .036$ \\
SES & -.15 & .069 & -.25 & .036 & & versus \\
& & & & & & $3: .001$ \\
7. Difficulty & -.03 & .03 & -.13 & .333 & .34 & versus \\
identifying feelings & & & & & & $4: .003$ \\
PTSD & -.01 & .005 & -.40 & .003 & & versus \\
& & & & & $5: 073$ \\
SES & -.13 & .07 & -.23 & .073 & & versus \\
& & & & & & $6: .333$ \\
\hline
\end{tabular}

and maltreatment throughout childhood and adulthood when compared to controls (see Fig. 3). Roughly half of the non-PTSD control group experienced one or more forms of interpersonal violence throughout their lifetime.
Table 2 shows group comparisons of severity of adult IPV in the current or most recent relationship with an intimate, maternal psychopathology, parenting stress, alexithymia, and maternal sensitivity.

Table 3 shows correlations of continuous measures of socioeconomic status, severity of adult IPV, maternal psychopathology, parenting stress, maternal difficulty in identifying feelings, and maternal sensitivity, controllingness, and unresponsiveness on the CARE-Index. In keeping with our first hypothesis, global severity of posttraumatic stress was significantly associated with mothers' difficulty in correctly identifying others' feelings. We had further hypothesized that maternal difficulty in identifying feelings would be most closely correlated with the severity of symptoms in the avoidance cluster of PTSD, as opposed to the re-experiencing and hyperarousal clusters. Difficulty identifying feelings was in fact most strongly correlated to CAPS hyperarousal and dissociative symptom severity, yet significantly associated with all PTSD symptom clusters: re-experiencing $\mathrm{r}=.34(p=.01) ;$ avoidance $\mathrm{r}=.36$ $(p=.006)$; hyperarousal $\mathrm{r}=.47(p<.001)$; dissociative symptoms $r=.49(p=.009)$. In agreement with our second hypothesis, maternal difficulty in correctly identifying others' feelings was negatively associated with maternal sensitivity.

Table 4 shows multiple linear regression models that tested the third hypothesis that maternal IPV- PTSD symptom severity and difficulty identifying feelings were additive predictors of decreased maternal sensitivity, after taking into account SES as a potential confounder. PTSD symptom severity and difficulty identifying feelings were each significantly associated with decreased maternal sensitivity when entered into the model after adjusting for
Fig. 2 All mothers with PTSD that were included had experienced interpersonal violence as adults, and a majority as children as well. Many of the non-PTSD controls also experienced interpersonal violence as children or adults

\section{Types of trauma suffered by group}

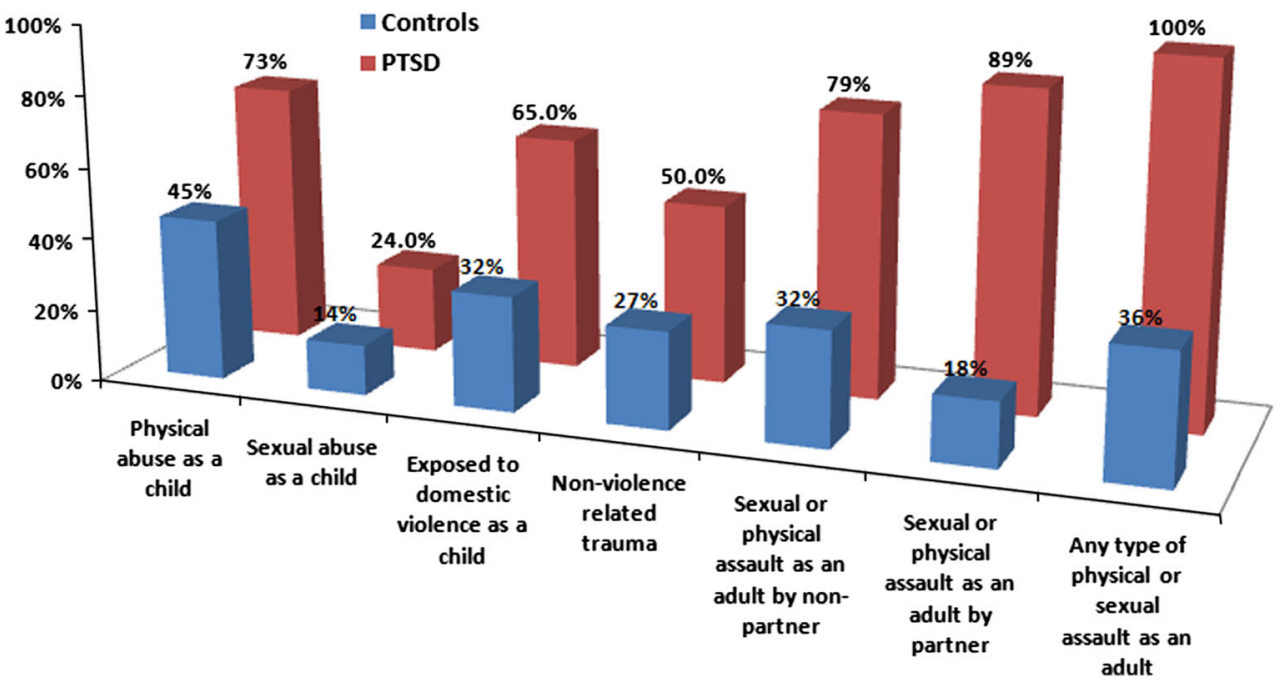




\section{Mothers without IPV-PTSD}

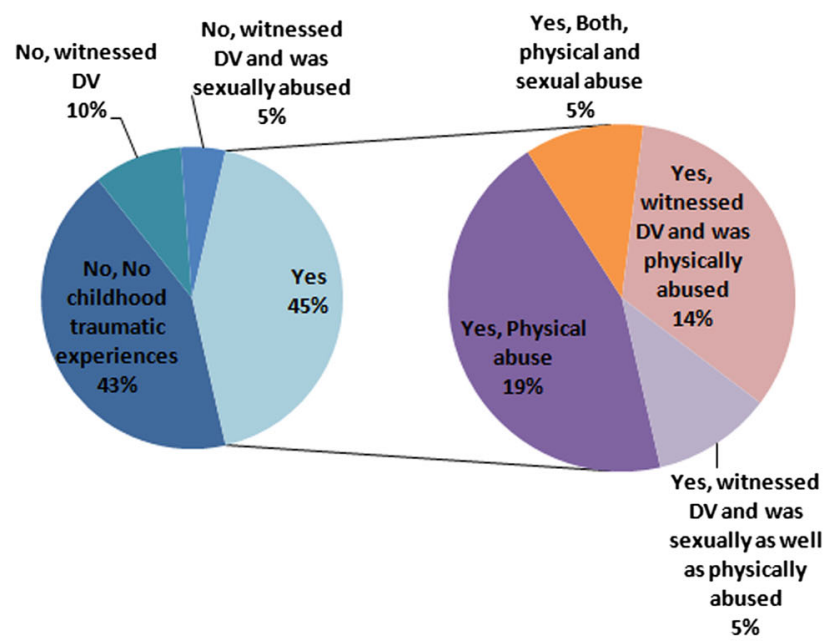

Mothers with IPV-PTSD

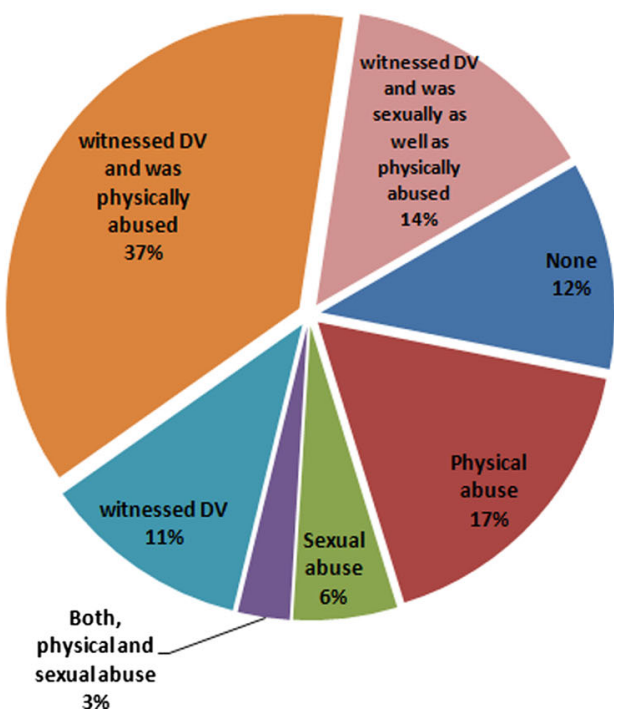

Fig. 3 Mothers with IPV-PTSD were more likely than controls to have experienced two or more forms of violence; whereas, non-PTSD controls were more likely to have experienced only a single form of violence if they had reported experiencing violence at all

SES. Taken together, the three variables accounted for $34 \%$ of the variance of maternal sensitivity. PTSD symptom severity, however, remained significantly associated with decreased maternal sensitivity in the full model, in contrast with maternal difficulty in identifying feelings and SES. The $F$ test applied to test the significance of change in R-square confirmed that maternal difficulty in identifying feelings fell short of contributing significantly to the model $(\mathrm{sig}=.097$ ). Although not displayed in Table 4, current maternal depressive symptom severity as measured on the BDI-II was added into a regression model with PTSD severity to predict maternal sensitivity. Maternal depressive symptom severity was neither significant $(p>.8)$ nor additive to the model. As seen in Table 3, the three aforementioned predictors as well as maternal depressive symptom severity were significantly correlated with each other, but collinearity diagnostics did not reveal any major estimation problem (all variance inflation factors $<2$ ).

\section{Discussion}

This study confirms a robust association between maternal difficulty in correctly identifying others' feelings, as measured by the subscale of the Toronto Alexithymia Scale-20 (TAS-20) of the same name and the severity of IPV-PTSD, as these two variables are negatively associated with maternal sensitivity on the CARE-Index. This TAS-20 subscale was positively correlated with IPV-PTSD symptoms in all symptom clusters, yet also robustly, with the dissociative symptom subscale of the Clinician Administered PTSD Scale (CAPS). The latter is consistent with the literature hypothesizing a relationship to emotional numbing among PTSD patients [17]. Maternal difficulty in identifying feelings had no significant relationship with IPV severity alone. The fact that IPV-PTSD but not IPV severity alone was associated with this TAS-20 subscale is consistent with the literature which suggests that this subscale is linked to the individual's psychobiological response to violence exposure and not to the experience of violence itself.

Several studies have pointed to the direct impact of violence-exposure on maternal mental representations, maternal sensitivity, and attachment security-linked variables without apparently having evaluated the subjects for PTSD and other forms of trauma-associated psychopathology $[44,45]$. The present findings lend support to the notion that violent events which mothers in this study experienced were not in and of themselves predictive of effects on their behavior as how their minds processed those events. PTSD is thus akin to a lens that colors and shapes the violence that mothers experienced [12, 41, 46]. And it is a specific lens that overrode the effects of maternal depressive symptom severity at the time of the observed mother-child interaction.

Findings supported the hypothesis that greater maternal difficulty in correctly identifying others' feelings was robustly and negatively associated with less maternal sensitivity. Yet this relationship paled when maternal PTSD severity was entered with it in a multiple linear regression model. One recent study showed that alexithymia as 
measured by the instrument used in this study (TAS-20) reflected a psychopathology-related state and avoidance of negative affects rather than an intrinsic or trait-related form of alexithymia [47].

Mothers, in any case, who have difficulty in correctly reading their toddlers' emotions or who exert avoidance or dissociation of negative feelings associated with traumatic memories of violence have been shown to have more hostile, intrusive behavior towards their young children $[48,49]$. In the present study, IPV-PTSD mothers as compared to non-PTSD mothers were indeed found to be more stressed as parents, more likely to become violent themselves, and more likely to harm themselves. Moreover, IPV-PTSD mothers displayed more controlling and unresponsive behavior on the CARE-Index than non-PTSD mothers. In terms of these findings pertinence to intergenerational transmission of trauma and violence, one study has shown that children of mothers with alexithymic difficulties, particularly male children, have been shown to develop aggressive behaviors [50].

We hypothesized that maternal difficulty identifying feelings in self and other would have an independent adverse effect on maternal sensitivity that would be additive to that of PTSD. Results did not support this hypothesis: when added to a model taking into account IPVPTSD symptom severity and SES, the contribution of maternal difficulty identifying feelings was not significant, with only a minor increase of the fraction of explained variance (from 32 to $34 \%$ ).

These results thus support that alexithymia in our sample is PTSD- and predominantly state- rather than traitrelated [47]. An alternative hypothesis based on the developmental psychology literature would be that maternal difficulties in reading, marking and responding contingently to the emotional communication of their young children might also be associated with a disturbance of attachment [51]. Child emotional abuse and neglect by the parent(s) within the context of a disturbed attachment, for example, have been associated with more severe alexithymia [52]. Indeed, many participant mothers who experienced interpersonal violence during adulthood had histories of childhood violence and maltreatment exposure that often, in turn, reflected most probably a disturbance of their primary attachment [53].

Trauma and attachment are therefore both independent yet overlapping variables to be considered in the context of early development and of the study of families with complex trauma histories across generations [54, 55]. Maternal sensitivity, a strong predictor of attachment security, was negatively associated with maternal difficulty in identifying feelings and maternal IPV-PTSD severity.

Similarly, maternal controlling and unresponsive behavior were also both associated with maternal IPV-
PTSD, but less with maternal alexithymia. Previous studies have found a relationship between the nature of maternal trauma involving physical violence and controlling, hostile, and intrusive caregiving [12, 41]. Unresponsive, withdrawing, and anxious-avoidant behavior was associated with higher levels of posttraumatic avoidance and dissociation $[41,56]$. Both behaviors, while very different, have been associated with increased oppositionality, dysregulated-aggressive and anxious behaviors among children $[57,58]$.

Maternal controlling behavior has been associated with child compulsive compliance and oppositionality [59]; and maternal unresponsive behavior has been associated with difficulty in maternal limit-setting, with subsequent fearful and aggressive behaviors [41]. Of additional interest, the relationships of these two atypical maternal behaviors was significant to maternal PTSD but not to depression. This likely points to the specific effects of maternal IPV-PTSD [60].

\section{Limitations}

One limitation of this study is that it was not possible given the cross-sectional rather than longitudinal nature of this study to understand the extent to which SES is a risk factor for maternal violence-exposure, PTSD, and alexithymia versus an effect of maladaptation resulting from chronic psychopathology [61, 62]. This study focused rather on the testing of a priori hypotheses that related the effects of maternal IPV-PTSD together with alexithymia on maternal behavior. Thus, the "chicken or egg" question of SES remained beyond the scope of this paper.

Another possible limitation was the use of the self-report version of the TAS-20 to measure alexithymia. Several articles have suggested that the validity of the TAS-20 subscale involving outwardly oriented thoughts has shown inadequate validity and reliability, therefore putting into question the overall score [63]. Yet, the subscale that we used to test our a priori hypotheses in the present studynamely, difficulties identifying feelings, showed excellent reliability in the same study. Given the complex prospect of asking someone who has difficulty in identifying and labeling emotions in self and other to complete a self-report questionnaire and to identify and label these difficulties has been cited as a drawback to the TAS-20 [64]. Thus the Toronto Structured Interview for Alexithymia (TSIA) has been used with increasing frequency and would be beneficial to subsequent study in this area [64, 65]. Another possible limitation is that we measured maternal sensitivity only during play that preceded the separation-reunion laboratory stressor. A next step would be to compare interactions prior to laboratory stressors, such as during free-play, with interactions following separation/noveltyexposure [56]. Future studies might also better define 
specific difficulties and confusion in identifying feelings in self and others via more precise, active social, cognitive and affective neuroscience-informed tasks, and to include more extensive data drawing directly from fathers.

\section{Summary}

This study tested the hypothesis that maternal difficulty in identifying emotions in self and other, as an important dimension of the construct of alexithymia, together with maternal IPV-PTSD, would increase the risk for disturbances in caregiving behavior that impact mutual emotion regulation within the mother-child relationship beyond the effect of either variable alone. Following maternal diagnostic interviews and administration of the Toronto Alexithymia Scale, 56 mothers and toddlers (ages 12-42 months) were filmed during free-play and separation/novelty-exposure. Observed maternal sensitivity was coded via the CARE-Index. Maternal IPV-PTSD severity, difficulty in identifying emotions, and lower socio-economic status (SES) were associated with less maternal sensitivity, and together accounted for more than one-third of the variance with respect to this dependent variable. Maternal IPV-PTSD severity, difficulty in identifying emotions, and lower socioeconomic status (SES) were also associated with greater maternal controlling and unresponsive behavior as consistent with previous findings [12, 41, 56, 57]. The findings of the present study suggest that clinical intervention with mothers who have IPV-PTSD and their very young children would best focus both on identifying, tolerating, and contextualizing IPV-associated emotions and memory traces in their children and themselves with the aid of an experienced clinician who models and supports mentalization [13, 24].

Acknowledgments This research was supported by the National Center of Competence in Research (NCCR) "SYNAPSY. The Synaptic Bases of Mental Diseases" financed by the Swiss National Science Foundation (No. 51AU40_125759), the Gertrude von Meissner Foundation, and la Fondation Prim'Enfance.

\section{References}

1. Breslau N, Kessler RC, Chilcoat HD, Schultz LR, Davis GC, Andreski P (1998) Trauma and posttraumatic stress disorder in the community: the 1996 Detroit Area Survey of Trauma. Arch Gen Psychiatry 55:626-632

2. Herman JL (1992) Complex PTSD: a syndrome in survivors of prolonged and repeated trauma. J Trauma Stress 5:377-391

3. van der Kolk BA, Roth S, Pelcovitz D, Sunday S, Spinazzola J (2005) Disorders of extreme stress: the empirical foundation of a complex adaptation to trauma. J Trauma Stress 18:389-399

4. Ditlevsen DN, Elklit A (2010) The combined effect of gender and age on post traumatic stress disorder: do men and women show differences in the lifespan distribution of the disorder? Ann Gen Psychiatry 9:32

5. Tremblay RE, Nagin DS, Seguin JR, Zoccolillo M, Zelazo PD, Boivin $M$ et al (2005) Physical aggression during early childhood: trajectories and predictors. Can Child Adolesc Psychiatr Rev 14:3-9

6. Fonagy P, Steele H, Steele M (1991) Maternal representations of attachment during pregnancy predict the organization of infantmother attachment at 1 year of age. Child Dev 62:891-905

7. Petitclerc A, Boivin M, Dionne G, Zoccolillo M, Tremblay RE (2009) Disregard for rules: the early development and predictors of a specific dimension of disruptive behavior disorders. J Child Psychol Psychiatry 50:1477-1484

8. Lyons-Ruth K, Bureau JF, Holmes B, Easterbrooks A, Brooks NH (2013) Borderline symptoms and suicidality/self-injury in late adolescence: prospectively observed relationship correlates in infancy and childhood. Psychiatry Res 206:273-281

9. Lenzi D, Trentini C, Pantano P, Macaluso E, Lenzi GL, Ammaniti M (2013) Attachment models affect brain responses in areas related to emotions and empathy in nulliparous women. Hum Brain Mapp 34:1399-1414

10. Schechter DS, Coots T, Zeanah $\mathrm{CH}$, Davies M, Coates SW, Trabka KA et al (2005) Maternal mental representations of the child in an inner-city clinical sample: violence-related posttraumatic stress and reflective functioning. Attach Hum Dev $7: 313-331$

11. Ainsworth MDS (1969) Scale 1: sensitivity versus insensitivity to the baby's signals, maternal sensitivity scales. Source mimeograph. Unpublished manuscript. The Baltimore Longitudinal Project, Johns Hopkins University, p. 2. http://www. psychology.sunysb.edu/attachment/measures/content/ainsworth_ scales.html

12. Lyons-Ruth K, Block D (1996) The disturbed caregiving system: relations among childhood trauma, maternal caregiving, and infant affect and attachment. Infant Ment Health J 17:257-275

13. Schechter DS, Rusconi S (2014) Understanding how traumatised mothers process their toddlers' affective communication under stress: towards preventive intervention for families at high risk for intergenerational violence. In: Emde R, Leuzinger-Bohleber $M$ (eds) Early parenting research and prevention of disorder: psychoanalytic research at interdisciplinary frontiers. Karnac Books, London, pp 90-118

14. Schechter DS, Myers MM, Brunelli SA, Coates SW, Zeanah CH, Davies $M$ et al (2006) Traumatized mothers can change their minds about their toddlers: understanding how a novel use of videofeedback supports positive change of maternal attributions. Infant Ment Health J 27:429-447

15. Frewen PA, Dozois DJ, Neufeld RW, Lanius RA (2008) Metaanalysis of alexithymia in posttraumatic stress disorder. J Trauma Stress 21:243-246

16. Sifneos PE (1973) The prevalence of 'alexithymic' characteristics in psychosomatic patients. Psychother Psychosom 22:255-262

17. Wang X, Chung MC, Hyland ME, Bahkeit M (2011) Posttraumatic stress disorder and psychiatric co-morbidity following stroke: the role of alexithymia. Psychiatry Res 188:51-57

18. Frewen PA, Pain C, Dozois DJ, Lanius RA (2006) Alexithymia in PTSD: psychometric and FMRI studies. Ann NY Acad Sci 1071:397-400

19. Fukunishi I, Sasaki K, Chishima Y, Anze M, Saijo M (1996) Emotional disturbances in trauma patients during the rehabilitation phase: studies of posttraumatic stress disorder and alexithymia. Gen Hosp Psychiatry 18:121-127

20. Fonzo GA, Simmons AN, Thorp SR, Norman SB, Paulus MP, Stein MB (2010) Exaggerated and disconnected insular-amygdalar blood oxygenation level-dependent response to threat- 
related emotional faces in women with intimate-partner violence posttraumatic stress disorder. Biol Psychiatry 68:433-441

21. Goerlich KS, Aleman A, Martens S (2012) The sound of feelings: electrophysiological responses to emotional speech in alexithymia. PLoS One 7:e36951

22. Gergely G, Watson JS (1996) The social biofeedback theory of parental affect-mirroring: the development of emotional selfawareness and self-control in infancy. Int $\mathrm{J}$ Psychoanal 77(Pt 6):1181-1212

23. Hinshaw-Fuselier S, Boris N, Zeanah CH (1999) Reactive attachment disorder in maltreated twins. Infant Ment Health $\mathbf{J}$ 20:42-59

24. Schechter DS, Moser DA, Reliford A, McCaw JE, Coates SW, Turner JB, Serpa SR, Willheim E (2014) Negative and distorted attributions towards child, self, and primary attachment figure among posttraumatically stressed mothers: what changes with clinician assisted videofeedback exposure sessions (CAVES). Child Psychiatry Hum Dev. doi:10.1007/s 10578-014-0447-5

25. Zeanah C, Larrieu JA, Heller SS, Vallier J (2000) Infant-parent relationship assessment. In: Press G, (ed) Handboof of infant mental health. New York, pp 222-235

26. Sancho Rossignol A, Lüthi Faivre F, Suardi F, Moser DA, Cordero MI, Rusconi Serpa S, Schechter DS (2010) Geneva sociodemographic Questionnaire (GSQ). Child and Adolescent Psychiatry Service, University of Geneva Hospitals, Geneva, Switzerland (unpublished)

27. First MB, Spitzer RL, Gibbon M, Williams JB (1995) Structured clinical interview for DSM-IV axis I disorders. American Psychiatric Press, Washington, DC

28. Largo RH, Pfister D, Molinari L, Kundu S, Lipp A, Duc G (1989) Significance of prenatal, perinatal and postnatal factors in the development of AGA preterm infants at five to 7 years. Dev Med Child Neurol 31:440-456

29. Marshall RD, Schneier FR, Fallon BA, Knight CB, Abbate LA, Goetz D et al (1998) An open trial of paroxetine in patients with noncombat-related, chronic posttraumatic stress disorder. J Clin Psychopharmacol 18:10-18

30. Kubany ES, Haynes SN, Leisen MB, Owens JA, Kaplan AS, Watson SB et al (2000) Development and preliminary validation of a brief broad-spectrum measure of trauma exposure: the Traumatic Life Events Questionnaire. Psychol Assess $12: 210-224$

31. Straus MA, Douglas EM (2004) A short form of the revised Conflict Tactics Scales, and typologies for severity and mutuality. Violence Vict 19:507-520

32. Blake DD, Weathers FW, Nagy LM, Kaloupek DG, Gusman FD, Charney DS et al (1995) The development of a clinicianadministered PTSD Scale. J Trauma Stress 8:75-90

33. Weathers FW, Keane TM, Davidson JR (2001) Clinicianadministered PTSD scale: a review of the first 10 years of research. Depress Anxiety 13:132-156

34. Blanchard EB, Jones-Alexander J, Buckley TC, Forneris CA (1996) Psychometric properties of the PTSD Checklist (PCL). Behav Res Ther 34:669-673

35. Beck AT, Steer RA, Brown GK (1996) Manual for the beck depression inventory-II. Psychological Corporation, San Antonio

36. Abidin R (1995) Parenting stress index. 3rd ed, Professional manual. Psychological Assessment Resources Inc., Lutz

37. Loas G, Parker JD, Otmani O, Verrier A, Fremaux D (1997) Confirmatory factor analysis of the French translation of the 20-item Toronto Alexithymia Scale. Percept Mot Skills 85:1018

38. Bagby RM, Taylor GJ, Parker JD (1994) The twenty-item Toronto Alexithymia Scale-II. Convergent, discriminant, and concurrent validity. J Psychosom Res 38:33-40

39. Crittenden PM (2006) CARE - Index: toddlers coding manual. Unpublished manual, available from the author
40. Crittenden PM, Bonvillian JD (1984) The relationship between maternal risk status and maternal sensitivity. Am J Orthopsychiatry 54:250-262

41. Schechter DS, Coates S, Kaminer T, Coots T, Zeanah C, Davies $M$ et al (2008) Distorded maternal mental representations and atypical behavior in a clinical sample of violence-exposed mothers and their toddlers. J Trauma Dissociation 9:123-147

42. Farnfield S, Hautamaki A, Norbech P, Sahhar N (2010) DMM assessments of attachment and adaptation: procedures, validity and utility. Clin Child Psychol Psychiatry 15:313-328

43. Kunster AK, Fegert JM, Ziegenhain U (2010) Assessing parentchild interaction in the preschool years: a pilot study on the psychometric properties of the toddler CARE-Index. Clin Child Psychol Psychiatry 15:379-389

44. Levendosky AA, Bogat GA, Huth-Bocks AC, Rosenblum K, von Eye A (2011) The effects of domestic violence on the stability of attachment from infancy to preschool. J Clin Child Adolesc Psychol 40:398-410

45. Miranda JK, de la Osa N, Granero R, Ezpeleta L (2013) Multiple mediators of the relationships among maternal childhood abuse, intimate partner violence, and offspring psychopathology. J Interpers Violence 28:2941-2965

46. Gustafsson HC, Cox MJ, Blair C (2012) Maternal parenting as a mediator of the relationship between intimate partner violence and effortful control. J Fam Psychol 26:115-123

47. Marchesi C, Ossola P, Tonna M, De Panfilis C (2014) The TAS20 more likely measures negative affects rather than alexithymia itself in patients with major depression, panic disorder, eating disorders and substance use disorders. Compr Psychiatry 55:972-978

48. McFarlane J, Symes L, Binder BK, Maddoux J, Paulson R (2014) Maternal-child dyads of functioning: the intergenerational impact of violence against women on children. Matern Child Health J. doi:10.1007/s10995-014-1473-4

49. Amos J, Furber G, Segal L (2011) Understanding maltreating mothers: a synthesis of relational trauma, attachment disorganization, structural dissociation of the personality, and experiential avoidance. J Trauma Dissociation 12:495-509

50. Bickett LR, Milich R, Brown RT (1996) Attributional styles of aggressive boys and their mothers. J Abnorm Child Psychol 24:457-472

51. Deborde AS, Miljkovitch R, Roy C, Dugre-Le Bigre C, PhamScottez A, Speranza M et al (2012) Alexithymia as a mediator between attachment and the development of borderline personality disorder in adolescence. J Pers Disord 26:676-688

52. Gulec MY, Altintas M, Inanc L, Bezgin CH, Koca EK, Gulec $\mathrm{H}$ (2013) Effects of childhood trauma on somatization in major depressive disorder: the role of alexithymia. J Affect Disord 146:137-141

53. Heller SS, Boris NW, Fuselier SH, Page T, Koren-Karie N, Miron D (2006) Reactive attachment disorder in maltreated twins follow-up: from 18 months to 8 years. Attach Hum Dev 8:63-86

54. Kuijpers KF, van der Knaap LM, Winkel FW (2012) Risk of revictimization of intimate partner violence: the role of attachment, anger and violent behavior of the victim. J Fam Violence 27:33-44

55. Perry R, Sullivan RM (2014) Neurobiology of attachment to an abusive caregiver: short-term benefits and long-term costs. Dev Psychobiol. doi:10.1002/dev.21219

56. Schechter DS, Willheim E, Hinojosa C, Scholfield-Kleinman K, Turner JB, McCaw J et al (2010) Subjective and objective measures of parent-child relationship dysfunction, child separation distress, and joint attention. Psychiatry 73:130-144

57. Cohen LR, Hien DA, Batchelder S (2008) The impact of cumulative maternal trauma and diagnosis on parenting behavior. Child Maltreat 13:27-38 
58. Schechter DS, Zygmunt A, Coates SW, Davies M, Trabka K, McCaw J et al (2007) Caregiver traumatization adversely impacts young children's mental representations on the MacArthur story stem battery. Attach Hum Dev 9:187-205

59. Crittenden PM, DiLalla DL (1988) Compulsive compliance: the development of an inhibitory coping strategy in infancy. J Abnorm Child Psychol 16:585-599

60. Schechter DS, Willheim E (2009) Disturbances of attachment and parental psychopathology in early childhood. Child Adolesc Psychiatr Clin N Am 18:665-686

61. Koenen KC (2007) Genetics of posttraumatic stress disorder: review and recommendations for future studies. J Trauma Stress 20:737-750

62. Koenen KC, Moffitt TE, Poulton R, Martin J, Caspi A (2007) Early childhood factors associated with the development of post- traumatic stress disorder: results from a longitudinal birth cohort. Psychol Med 37:181-192

63. Kooiman CG, Spinhoven P, Trijsburg RW (2002) The assessment of alexithymia: a critical review of the literature and a psychometric study of the Toronto Alexithymia Scale-20. J Psychosom Res 53:1083-1090

64. Lichev V, Rufer M, Rosenberg N, Ihme K, Grabe HJ, Kugel H et al (2014) Assessing alexithymia and emotional awareness: relations between measures in a German non-clinical sample. Compr Psychiatry 55:952-959

65. Caretti V, Porcelli P, Solano L, Schimmenti A, Bagby RM, Taylor GJ (2011) Reliability and validity of the Toronto structured interview for Alexithymia in a mixed clinical and nonclinical sample from Italy. Psychiatry Res 187:432-436 